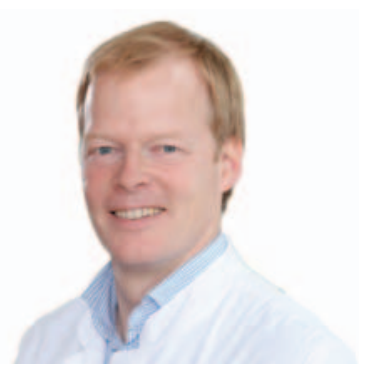

\title{
AMD-Patienten müssen dauerhaft und regelmäßig kontrolliert werden
}

Prof. Dr. Nicolas Feltgen

Universitätsmedizin Göttingen, Abteilung für Augenheilkunde

Abstractübersetzung aus Wolf S, Bandello F, Loewenstein A, Slakter J, Katz T, Sowade O, Korobelnik J-F: Baseline characteristics of the fellow eye in patients with neovascular age-related macular degeneration: post hoc analysis of the VIEW studies. Ophthalmologica 2016;236:95-99.

\section{Merkmale des Partnerauges bei Patienten mit neovaskulärer altersbedingter Makuladegeneration zu Studienbeginn: Post-hoc-Analyse der VIEW-Studien}

\section{Schlüsselwörter}

Anti-VEGF-Wirkstoffe · Bestkorrigierter Visus .

Bilaterale Erkrankungen · Choroidale Neovaskularisation .

Partnerauge · Intravitreales Aflibercept .

Neovaskuläre altersbedingte Makuladegeneration .

Gepoolte Analyse · Ranibizumab

\section{Zusammenfassung}

Ziel: Ziel war es, die bei Studienbeginn vorliegenden Merkmale des Partnerauges bei Patienten mit neovaskulärer altersbedingter Makuladegeneration (nAMD) zu beschreiben.

Methoden: Es wurde eine gepoolte Post-hoc-Analyse von nAMDPatienten durchgeführt, die an den VIEW-Studien teilnahmen. In den VIEW-Studien wurde intravitreal injiziertes Aflibercept (monatlich oder alle 2 Monate nach 3 Injektionen $1 \times$ pro Monat) mit monatlich verabreichtem Ranibizumab verglichen. Bewertet wurden der Status der choroidalen Neovaskularisation (CNV) des Partnerauges und der bestkorrigierte Visus (BCVA) bei Studienbeginn sowie der Linsenstatus beider Augen. In weiteren Analysen wurde das Vorhandensein von Drusen und Pigment im Partnerauge bewertet.

Ergebnisse: Der Vergleich beider Augen ergab, dass der BCVA zu Studienbeginn bei 23,8\% der Partneraugen und bei 75,2\% der betroffenen Augen schlechter war. Der Linsenstatus der Partner- und betroffenen Augen war vergleichbar. Der Visus des betroffenen Auges und des Partnerauges zu Studienbeginn wurden nicht korreliert. Die meisten Partneraugen wiesen Anzeichen von AMD im Frühstadium auf, wobei 34,6\% $(n=843)$ der Partneraugen eine Vernarbung zeigten.

Schlussfolgerungen: Bei den Patienten in den VIEW-Studien zeigten sich in den meisten Partneraugen Hinweise auf AMD. Dies unterstreicht die Wichtigkeit, beide Augen zu untersuchen, gefolgt von einer engmaschigen Nachsorge, um CNV möglichst frühzeitig zu erkennen und zu behandeln.

(c) 2017 S. Karger GmbH, Freiburg

\section{KARGER}

() 2017 S. Karger GmbH, Freiburg 


\section{Transfer in die Praxis}

\section{Hintergrund}

Das Risiko von Patienten mit einer altersabhängigen Makuladegeneration (AMD) auch am zweiten Auge eine chorioidale Neovaskularisation (CNV) zu entwickeln ist relativ hoch und wird in der Literatur mit 4-19\% beschrieben. Für uns behandelnde Augenärzte ist die Konsequenz aus diesem doch recht hohen Risiko, so oft als möglich auch das zweite Auge mit zu untersuchen. Auch wenn eine längere Injektionsserie mit einem fixen Injektionsintervall erfolgt, wie z.B. eine zweimonatliche Injektion innerhalb des ersten Behandlungsjahres, sollte das Partnerauge bei jeder Visite mituntersucht werden.

Wie häufig und in welcher Ausprägung besteht aber bei Erstvorstellung am Partnerauge bereits eine AMD, und führt eine Vorerkrankung am ersten Auge zu einer frühen Vorstellung am zweiten Auge?

\section{Studienergebnisse}

Diese Frage hat sich das Autorenteam um Wolf et al. gestellt und konnte sie mithilfe einer Sekundäranalyse anhand der umfangreichen VIEW-Studiendaten beantworten. In die beiden identischen Behandlungsstudien wurden zwischen 2007 und 2010 weltweit insgesamt 2412 AMD Patienten mit einer CNV eingeschlossen.

\section{Das Partnerauge zeigt nahezu immer AMD-typische Verände-} rungen, in einem Drittel sind das fortgeschrittene Stadien In nahezu allen Fällen war auch das zweite Auge, das nicht in die Studie eingeschlossen wurde, von einer AMD in unterschiedlichem Ausmaß betroffen. Lediglich 10\% aller Augen hatten keine AMD-typischen Veränderungen. Der mittlere Visus am Partnerauge war zwar etwas besser (Fernvisus $(F V)=0,4$ ) als am Studienauge ( $F V=0,25)$, die Streubreite der Visuswerte war am Partnerauge aber deutlich breiter als am Studienauge. Diese Beobachtung ist durch die Studieneinschlusskriterien am behandelnden Auge (Visus zwischen 0,063 und 0,63) zu erklären. Ernüchternd ist aber die Tatsache, dass eine Sehschärfe von unter 0,1 am Partnerauge bei mehr Patienten festgestellt wurde, als dies am Studienauge der Fall war. Ebenso war bei knapp 35\% aller Patienten am Partnerauge bereits eine AMD-typische Vernarbung sichtbar.

\section{Kritik und Fazit für die Praxis}

\section{AMD-Patienten müssen dauerhaft und regelmäßig kontrolliert werden}

Damit können wir nicht mehr davon ausgehen, dass Patienten, die bereits einen irreversiblen Visusverlust am ersten Auge hatten, bei Veränderungen des zweiten Auges automatisch früher den Augenarzt aufsuchen. Das wiederum verpflichtet den betreuenden Ophthalmologen, auch nach abgeschlossener Behandlung am ersten Auge dauerhafte und regelmäßige Kontrollen zu empfehlen.

\section{Untersuchungen in Zeiten des therapeutischen Umbruchs könnten die Datenlage beeinflussen}

Bei der Beurteilung der Daten muss man sich natürlich fragen, ob die in dem Zeitraum zwischen 2007 und 2010 eingeschlos- senen Patienten ein repräsentatives Kollektiv darstellen. Im Jahr 2007 wurde Ranibizumab für die Therapie der CNV im Rahmen einer AMD zugelassen, zuvor stand mit Bevacizumab bereits eine potente Substanz zur Verfügung. Trotzdem war es die Zeit des Umbruchs vom therapeutischen Nihilismus zur ersten visusverbessernden oder zumindest stabilisierenden Behandlung. Es ist davon auszugehen, dass das Bewusstsein der Bevölkerung für diese bedeutsame Erkrankung durch vielfache Informationsmaßnahmen zugenommen hat. Deshalb ist es denkbar, dass eine vergleichbare Studie weniger fortgeschrittene Befunde am ersten Auge zeigt, als dies noch in den VIEWStudien der Fall war.

\section{Was ist zu tun?}

Da die AMD weit verbreitet ist und für uns Augenärzte ein sehr vertrautes Krankheitsbild darstellt, besteht die Gefahr, dieses Wissen auch in der Bevölkerung stillschweigend vorauszusetzen. In einer jüngsten Umfrage zu wichtigen ophthalmologischen Notfällen zeigten sich hingegen erschreckende Daten. Nur 28\% aller Befragten Patienten in einer Notaufnahme eines New Yorker Krankenhauses kannten die Ablatio retinae, nur 15\% den Begriff des Glaukomanfalls, und jeweils 5\% die Symptome einer Arteriitis temporalis oder eines Zentralarterienverschlusses [1]. Dabei bewirkt konsequente Information dauerhaft tatsächlich eine Verbesserung. In einer AMD-Umfrage aus dem Jahr 2014 in Singapur wussten im Jahr 2006 lediglich 7,3\% mit der AMD etwas anzufangen, im Jahr 2011 waren es bereits 28,1\% [2].

Die Ergebnisse bei der Therapie der CNV im Rahmen einer AMD korrelieren stark mit dem Ausgangsvisus. Es ist deshalb elementar für ein gutes Abschlussergebnis, möglichst frühzeitig zu therapieren. Dazu müssen wir als Augenärzte die Bevölkerung verstärkt informieren und über die typischen Symptome aufklären. Auch nach Diagnosestellung ist die konsequente und dauerhafte Kontrolle ein wesentlicher Bestandteil der erfolgreichen Behandlung.

\section{Disclosure Statement}

Hiermit erkläre ich, dass keine Interessenskonflikte in Bezug auf den vorliegenden Kommentar bestehen.

\section{Literatur}

1 Uhr JH, Mishra K, Wei C, Wu AY: Awareness and knowledge of emergent ophthalmic disease among patients in an internal medicine clinic. JAMA Ophthalmol 2016;134:424-431.

2 Sanjay S, Chin YC, Teo HT, et al.: A follow-up survey on the knowledge of age-related macular degeneration and its risk factors among Singapore residents after 5 years of nation-wide awareness campaigns. Ophthalmic Epidemiol 2014;21:230-236.

Kontaktadresse: Prof. Dr. Nicolas Feltgen, Universitätsmedizin Göttingen, Abteilung für Augenheilkunde, Robert-Koch-Straße 40, 37075 Göttingen, Deutschland, nicolas.feltgen@med.uni-goettingen.de 